\title{
Rapid Detection of Infestation of Apple Fruits by the Peach Fruit Moth, Carposina sasakii Matsumura, Larvae Using a 0.2-T Dedicated Magnetic Resonance Imaging Apparatus
}

\author{
Tomoyuki Haishi · Hiroshi Koizumi • \\ Tomonori Arai $\cdot$ Mika Koizumi $\cdot$ Hiromi Kano
}

Received: 4 November 2010/Revised: 8 February 2011/Published online: 22 April 2011

(C) The Author(s) 2011. This article is published with open access at Springerlink.com

\begin{abstract}
Infestation of harvested apple fruits by the peach fruit moth (Carposina sasakii Matsumura) was studied using a dedicated magnetic resonance imaging (MRI) apparatus equipped with a $0.2-\mathrm{T}$ permanent magnet. Infested holes on the three-dimensional (3-D) images tracked ecological movements of peach fruit moth larvae within the food fruits, and thus in their natural habitat. Sensitive short solenoid coil and surface coil detectors were devised to shorten measurement times. The short solenoid coil detected infestation holes at a rate of $6.4 \mathrm{~s}$ per image by the single-slice 2-D measurement. The multi-slice 2-D measurement provided six slice images of a fruit within 2 min taken by the two detectors. These results indicate that the 0.2-T MRI apparatus allows one to distinguish sound fruits from infested ones, and also as a means for plant protection and the preservation of natural ecological systems in foreign trade.
\end{abstract}

\section{T. Haishi $(\bowtie)$}

MR-Technology, Inc., 2-1-6 Sengen, Tsukuba, Ibaraki 305-0047, Japan

e-mail: haishi@mrtechnology.co.jp

H. Koizumi

Faculty of Science and Engineering, Waseda University,

2-2 Wakamatsucho, Shinjyuku, Tokyo 162-8480, Japan

T. Arai

National Institute of Fruit Tree Science, National Agriculture and Food Research Organization, 92-24 Nabeyashiki, Kuriyagawa, Morioka, Iwate 020-0123, Japan

\section{Koizumi}

Research Institute for Science and Engineering, Waseda University,

2-2 Wakamatsucho, Shinjyuku, Tokyo 162-8480, Japan

H. Kano

Oak-Hill Georgic Patch-Work Laboratory, 4-13-10 Miyamoto,

Funabashi, Chiba 273-0003, Japan 


\section{Introduction}

The peach fruit moth (Carposina sasakii Matsumura) is an insect that damages apple fruits. The entrances of the larvae into the fruits are very small, and the moth tends not to put out excreta from the fruits. At the same time, ecological effects of the larvae in the food fruits have not been defined. It is difficult to identify an infestation from outside the fruit. Infestation by larvae of the insect deteriorates the quality of products and leads to a loss of reliability in production areas. Pesticides are used periodically, based on ecological studies of the moth [1, 2], to avoid severe damage, and fruits are carefully examined by the human eye during harvesting and cargo booking. For this reason, infested fruits are seldom sold on the Japanese market. But, to avoid the larvae infestation completely, a new method is needed. From the standpoints of better understanding of larval ecological effects, plant protection and preservation of natural ecological systems in the export of apples, noninvasive detection of the moth needs to be devised.

The internal condition of apples (e.g., water core [3, 4], mealiness [5] and bruising [6] has been examined by magnetic resonance imaging (MRI). MRI has been used to observe the developmental changes of the desert locust (Schistocerca gregaria (Forskal)) embryo [7] and lepidopteran pupae (Pieris brassicae and Graphiphora augur) [8]; the morphology of the diving beetle (Dytiscus marginalis) [9], common wasp (Vespula vulgaris) queen and large ant (Dinoponera quadriceps) worker [10]; the host-parasitoid interaction of the seven-spot ladybird (Coccinella 7-punctata (Coleoptera: Coccinellidae)) infected with small parasitic wasps [Dinocampus coccinellae (Hymenoptera: Braconidae)] [11]; and the dynamics of circulatory, respiratory and digestive systems in pupae of the tobacco hornworm [Manduca sexta L. (Lepidoptera: Sphingidae)] [12]. Thus, MRI is useful for investigating the morphology, physiology and host-parasite interaction of insects $[10,13]$.

On the basis of our previous findings, the infestation of young apple fruits by peach fruit moth larvae can be visualized using a dedicated MRI apparatus equipped with a 1-T field strength permanent magnet [14], the field of view (FOV) of which is restricted within $30 \mathrm{~mm}$, though resolution is high [15]. However, that apparatus could not be used for grown or harvested fruits, which were the primary targets for larval growth and the distribution media of the moth to foreign areas. Therefore, we constructed another MRI apparatus equipped with a 0.2-T permanent magnet and a 160-mm pole air gap, and demonstrated that mature larvae could be detected and infestation of the fruits could be tracked by the new apparatus [16]. This device has the following operational benefits: it is small, lightweight and maintenance free. It requires a low level of electric power (e.g., AC $100 \mathrm{~V}$ at $15 \mathrm{~A}$ ). It is easy to operate and can be sited conveniently by researchers.

In this investigation, we first confirm some of the results from our previous paper [16] that MRI is a useful modality to detect infestation of apple fruits by peach fruit moth larvae. We then determine how quickly the infestation could be detected using the 0.2-T dedicated MRI apparatus, which has a relatively low image resolution and a limited sensitivity due to low magnetic field strength. Traces of infestation are mapped out in three-dimensional (3-D) measurements using a 3-D gradient echo 
method, while two sensitive detectors specified for 2-D measurements with 2-D gradient echo methods are devised for reducing measurement times. This approach allows the apparatus to observe natural ecological systems, examining infested fruits routinely as a stand-alone device or as a sensor for detecting infestation within an automated system.

\section{Materials and Methods}

\subsection{Infested Apple Fruits}

Paraffin papers with the eggs of peach fruit moth, Carposina sasakii Matsumura (Lepidoptera: Carposinidae), were attached to 'Fuji' apple (Malus domestica Borkhausen) cultivars harvested in November 2008 in a nonpesticide field of the National Institute of Fruit Tree Science, Apple Research Station (Morioka, Japan), and the apples were incubated in a room with 16-h light and 8-h dark regime at $20^{\circ} \mathrm{C}$. Larvae hatched on 20 and 21 November and 12 December 2008, and then entered into the apple fruits. Measurements were carried out on 11 and 15 December 2008 , for the earlier fruit infestations, and on the 7th, 14th and 21st day after hatching for the latter case. $T_{1}$ and $T_{2}$ relaxation times of a whole apple measured on the 0.2-T apparatus were about 800 and $300 \mathrm{~ms}$ at $20^{\circ} \mathrm{C}$, respectively.

\subsection{MRI Apparatus}

The dedicated MRI apparatus equipped with a permanent magnet was constructed and modified for studying infestation of grown apple fruits. The magnet (Fig. 1a) had a field strength of $0.2-\mathrm{T}(8.9 \mathrm{MHz})$, dimensions of $50 \mathrm{~cm}(W) \times 50 \mathrm{~cm}$ $(D) \times 75 \mathrm{~cm}(H)$, had a $160-\mathrm{mm}$ pole gap and weighed $500 \mathrm{~kg}$. The magnet, which was made of $\mathrm{Nd}-\mathrm{Fe}-\mathrm{B}$ blocks capable of generating a strong magnetic field (NEOMAX Engineering Co. Ltd., Gunma, Japan), was specially designed for the apparatus. The poles (Fig. 1b, $b$ and $c$ ) were sustained by iron yokes (Fig. 1b, $d$ ), which were joined by two iron bridges at the bottom (Fig. 1b, f). Therefore, the direction of the magnetic field was horizontal (denoted as the $Z$-axis). The temperature around the whole magnet was maintained at $29.0^{\circ} \mathrm{C}$ with a controller to prevent any shift of resonating radio frequency and to stabilize the signal intensity against the influence of ambient temperature [17] during an imaging experiment. Since there was a wide air gap $(160 \mathrm{~mm})$ between the poles exposed to ambient air, the temperature in the measurement cell was near room temperature. The magnet was equipped with gradient coils [Fig. 1b, $e$; gradient efficiency of GX/GY/ $\mathrm{GZ}=1.3 / 1.3 / 2.7(\mathrm{mT} / \mathrm{m}) / \mathrm{A}]$, which collaterally functioned as the first-order SHIM (a system shimming the magnetic field). The apparatus had a separate MRI spectrometer (Fig. 1c), which had the dimensions of $60 \mathrm{~cm}(W) \times 60 \mathrm{~cm}$ $(D) \times 80 \mathrm{~cm}(H)$ and weighed $100 \mathrm{~kg}$, with a rack-mounted personal computer running the Windows XP-SP3 operating system (Microsoft Co., Tokyo, Japan), with a console's display, mouse and keyboard. The system was designed to be space saving as it was set in a space of $2 \mathrm{~m}^{2}$ [16]. 

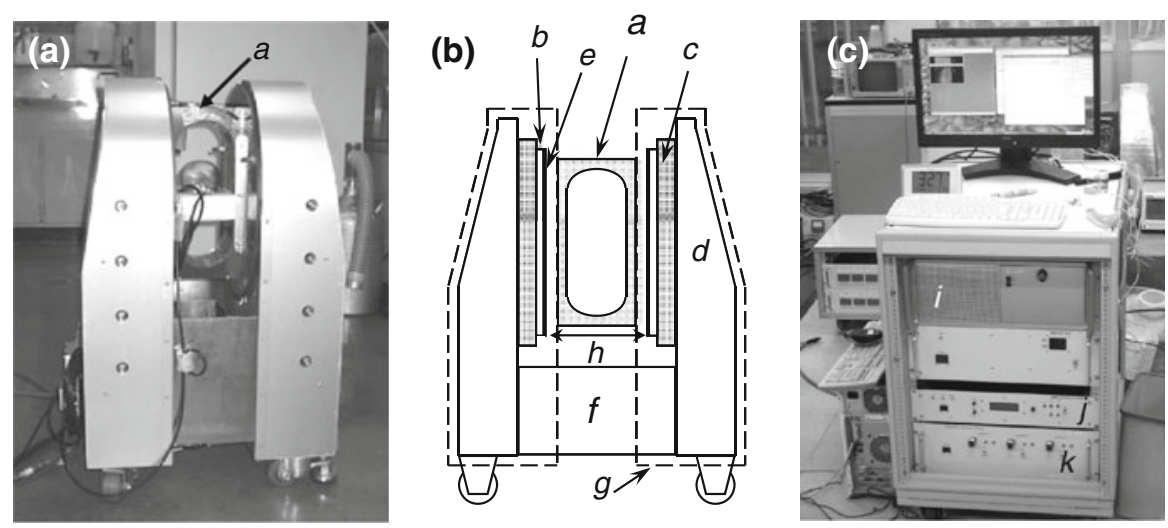

Fig. 1 Dedicated MRI. a Permanent magnet $\left(50 \times 50 \mathrm{~cm}^{2}, 75 \mathrm{~cm}\right.$ high, and $\left.500 \mathrm{~kg}\right)$ with magnetic field strength of $0.2 \mathrm{~T}$ and air gap of $160 \mathrm{~mm}$. A detector with housing for shielding from external radiofrequency (rf) noise is indicated as a. $a$. b Block diagram illustrating the relationship between magnet components and the detector: $a$ detector, $b$ pole piece, $c$ magnetic metals, $d$ yoke, $e$ gradient coil, $f$ bridge joining two yokes, $g$ outside cover, $h 160-\mathrm{mm}$ pole gap. c MRI spectrometer $\left(60 \times 60 \mathrm{~cm}^{2}, 80 \mathrm{~cm}\right.$ high, and $100 \mathrm{~kg}$ ) with a rack-mounted computer with a Windows XP-SP3 operating system. The console display, mouse, and keyboard were placed on the top plate of the spectrometer. $i$ Rack-mounted computer, $j$ rf transceiver, $k$ gradient unit

\subsection{Detectors}

A detector was set between poles of the magnet (Fig. 1a, b; a) and could be freely exchanged with handmade ones. Three different types of detectors were used. The first, a seven-turn 110-mm diameter solenoid coil (110-mm Sol.) detector, $75 \mathrm{~mm}$ in length (Fig. 2a, d), was used for 3-D measurements. It had a specific housing for shielding against external radio-frequency (rf) noise. The coil was shorter than its diameter to obtain strong resonance, and the $Q$ value was 150 (at $-3 \mathrm{~dB}$ ) when impedance was adjusted to $50 \Omega$.

The second and third types of detectors for 2-D measurements used a short solenoid coil (Short Sol.) detector that was $94 \mathrm{~mm}$ in diameter and $35 \mathrm{~mm}$ in length and comprised four turns (Fig. 2b, e), and a surface coil (Surface C.) detector of four loops, $75 \mathrm{~mm}$ in inner diameter and $5 \mathrm{~mm}$ thick (Fig. 2c, f), respectively. These were specially designed for improving sensitivity around the imaging slice in 2-D measurements by narrowing the diameter and shortening the length of the coils, based on the results of Hoult and Richards [18]. The 2-D detectors were placed in a common oval housing and shielded from external $\mathrm{rf}$ noise. The former had a $Q$ value of 170 (at $-3 \mathrm{~dB}$ ), and the latter had a $Q$ value of 155 (at $-3 \mathrm{~dB}$ ) when impedance was adjusted to $50 \Omega$.

Figure 3a, b shows 1-D MRI signal intensity profiles and signal-to-noise ratio of a bottle water phantom $\left(67 \mathrm{~mm} \phi \times 120 \mathrm{~mm}\right.$ length, $\mathrm{CuSO}_{4}$-doped water, $T_{1} / T_{2}=$ $35 \mathrm{~ms} / 35 \mathrm{~ms}$ ) measured with the three coils and acquired using a 2-D gradient echo sequence (repetition time (TR)/echo time (TE)/flip angle (FA) $=500 \mathrm{~ms} / 7 \mathrm{~ms} / 90^{\circ}$, 


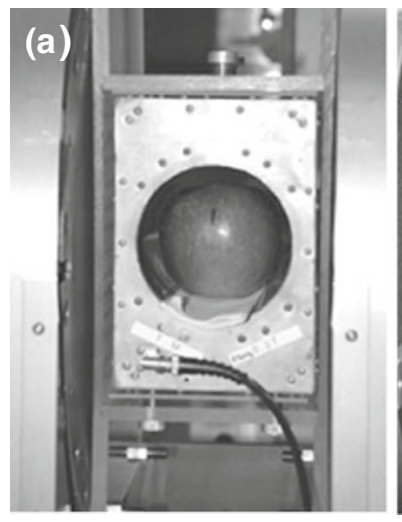

(d)

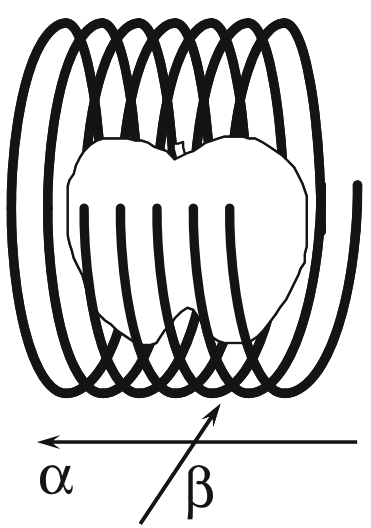

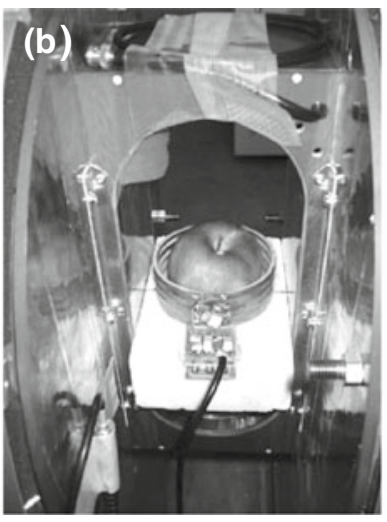

(e)

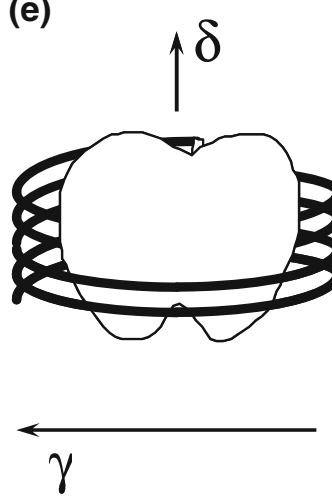

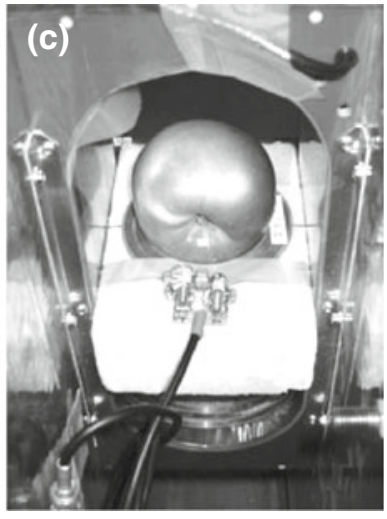

(f)

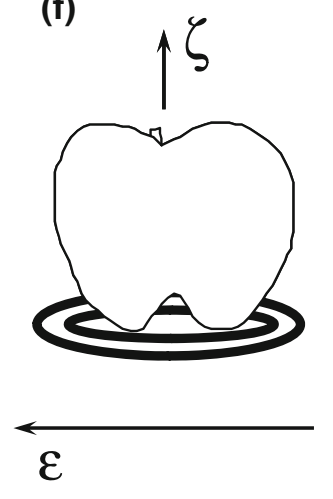

Fig. 2 Detector and coil layouts devised for the 0.2-T MRI apparatus. a and d 110-mm solenoid coil detector with a length of $75 \mathrm{~mm}$. b and e Short solenoid coil detector, $94 \mathrm{~mm}$ in diameter and $35 \mathrm{~mm}$ long. $\mathbf{c}$ and $\mathbf{f}$ Surface coil detector, $75 \mathrm{~mm}$ in inner diameter and $5 \mathrm{~mm}$ thick. $\mathbf{b}$ and $\mathbf{c}$ were developed to increase the sensitivity of the measurement of 2-D images and placed in a common oval housing for shielding from external rf noise. Arrows labeled from $\alpha$ to $\zeta$ indicate coil axes or slice directions

$256 \times 128$ matrix, $10 \mathrm{~mm}$ slice thickness) and a 2-D spin echo sequence (TR/ $\mathrm{TE}=500 \mathrm{~ms} / 9 \mathrm{~ms}, 256 \times 128$ matrix, $10 \mathrm{~mm}$ slice thickness). These 1 -D profiles were abstracted from the 2-D images and all involved the MRI signal intensity crossing each coil's center. These profiles were acquired with the same receiver gain so that the coil's sensitivities of spatial MRI signals were comparable in Fig. 3a, b. The excitation rf powers for $90^{\circ}$ and $180^{\circ}$ pulses were adjusted experimentally for each coil's center individually. The gradient echo method achieves almost twice better MRI signal intensity of the water phantom than the 2-D spin echo method for all three coils. Figure 3 shows that the short solenoid coil has better sensitivity than the other two coils and is suitable for 2-D imaging around the coil's center. The surface coil received the best signal intensity at the coil's center, but had the worst nonuniform signal profiles in the coil's diameter and along the axis of around the three coils. This nonuniform profile of the surface coil caused difficulties in 
(a)

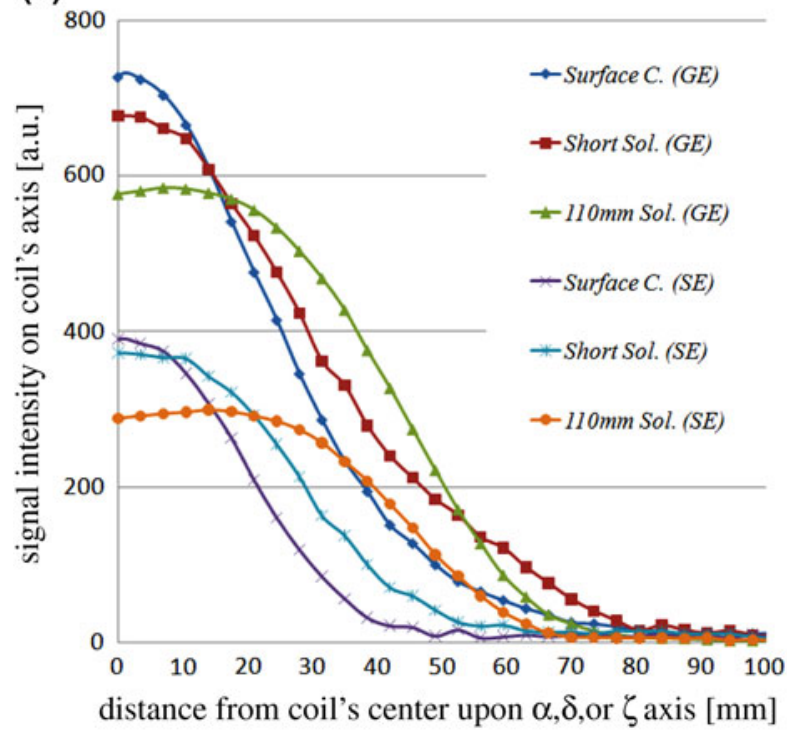

(b)

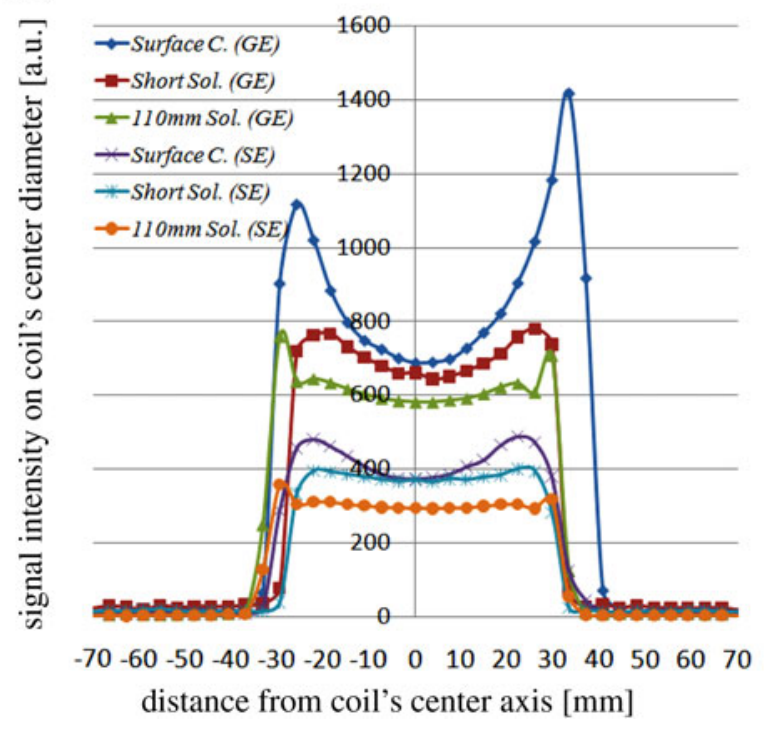

Fig. 3 1-D sensitivity profiles on two center axes for the three coil detectors. Vertical axis shows the signal intensity from a bottle water phantom $\left(\mathrm{CuSO}_{4}\right.$ doped, $\left.T_{1} / T_{2}=35 \mathrm{~ms} / 35 \mathrm{~ms}\right)$. Horizontal axis shows a the distance from the coil's center upon $\alpha-, \delta$-, or $\zeta$ - axis $(\mathrm{mm})$ or $\mathbf{b}$ the distance from the coil's center axis (mm). Each profile crossing the coil's center is abstracted from the 2-D image. 110-mm Sol. is a $110-\mathrm{mm}$ solenoid coil detector, $75 \mathrm{~mm}$ long. Short Sol. is a short solenoid coil detector, $94 \mathrm{~mm}$ in diameter and $35 \mathrm{~mm}$ long. Surface $C$. is a surface coil detector, $75 \mathrm{~mm}$ in inner diameter and $5 \mathrm{~mm}$ thick. $S E$ is a 2-D spin echo sequence (TR/TE $=500 \mathrm{~ms} / 9 \mathrm{~ms}, 256 \times 128$ matrix). $G E$ is a 2-D gradient echo sequence $\left(\mathrm{TR} / \mathrm{TE} / \mathrm{FA}=500 \mathrm{~ms} / 7 \mathrm{~ms} / 90^{\circ}, 256 \times 128\right.$ matrix) (color figure online) 
adjusting flip angles for gradient echo sequences and a signal intensity gradation in MR images. The 110-mm solenoid coil had the most flat signal profile, and thus was suitable for 3-D imaging of a whole apple, but detected less MRI signal at the coil's center than the other two coils.

\subsection{Measurements}

Table 1 summarizes parameters for imaging sequences discussed in this study. The progression of infestation was traced by the 3-D gradient echo method using the 110-mm solenoid coil detector (Fig. 4). TR and TE were 100 and $5 \mathrm{~ms}$, respectively, and the flip angle was $90^{\circ}$. The magnitudes of the gradient magnetic field were $\mathrm{G}($ readout $)=9.8 \mathrm{mT} / \mathrm{m}$, the maximum $\mathrm{G}(2-\mathrm{D}$ phase encode $)=8.4 \mathrm{mT} /$ $\mathrm{m}$, and the maximum $\mathrm{G}(3-\mathrm{D}$ phase encode $)=4.2 \mathrm{mT} / \mathrm{m}$. Data were obtained using a $256 \times 128 \times 64$ imaging matrix, and its signal accumulation was two times in each 2-D phase encoding. Total imaging time for 3-D data was $27 \mathrm{~min}$. The image was reconstructed to a $256 \times 256 \times 256$ matrix using a zero-filled Fourier interpolation method, and the resulting nominal isotropic spatial resolution was $430 \mu \mathrm{m}$. The FOV was about $11 \times 11 \times 11 \mathrm{~cm}$. For final infestation assessment, images were further processed by using ImageJ program [a public domain JAVA image processing program (Ver. 1.42); available on the Internet at http://rsb.info.nih.gov/ij/].

Rapid detection of infestation was attempted by either the single-slice 2-D gradient echo method with thickness of $16 \mathrm{~mm}$ (readout gradient $=9.8 \mathrm{mT} / \mathrm{m}$, slicing gradient $=11.7 \mathrm{mT} / \mathrm{m}, \pm 4 \mathrm{kHz}$ sinc-pulse, Figs. $5-7$ ) or the multi-slice $2-\mathrm{D}$ gradient echo method with thickness of $10 \mathrm{~mm}$ (readout gradient $=4.9 \mathrm{mT} / \mathrm{m}$, slicing gradient $=4.7 \mathrm{mT} / \mathrm{m}, \pm 1 \mathrm{kHz}$ sinc-pulse, Fig. 8) using all three detectors for a field of view $(\mathrm{FOV})$ of $11 \times 11 \mathrm{~cm}$. The maximum magnitude of the phase gradient magnetic field was $8.4 \mathrm{mT} / \mathrm{m}$. The flip angle of the excitation radio frequency was $30^{\circ}$ for the $110-\mathrm{mm}$ solenoid coil detector and $5^{\circ}$ for the coil's centers of 2-D specified detectors, which were suitable to induce strong contrasts. Three vertical 2-D images $(128 \times 128$ matrix, Fig. 5) acquired with the three coils were measured to survey the detectability of the apple larvae and gradations of MRI signal intensity. The effect of changing the number of signal accumulations $(16,8,4$ and 2 times, Fig. 6) or 2-D phase encode (128, 64 and 32 steps, Fig. 7) to the 2-D apple images was investigated with TR, TE and the same readout sampling number of $100 \mathrm{~ms}, 5 \mathrm{~ms}$ and 128 points, respectively. The resulting nominal planar resolution was $860 \mu \mathrm{m}$.

As for the 2-D multi-slice gradient echo measurements (Fig. 8), the readout gradient was $4.9 \mathrm{mT} / \mathrm{m}$ and the maximum magnitude of the phase-encoding gradient was $8.4 \mathrm{mT} / \mathrm{m}$. TR and TE were 200 and $7 \mathrm{~ms}$, and flip angles (110-mm Sol. $=30^{\circ}$, Short Sol. $=5^{\circ}$, and Surface Coil $=5^{\circ}$ ) were adjusted experimentally at the centers of the three coils. A $256 \times 128$ matrix for a field of view (FOV) of $11 \times 11 \mathrm{~cm}$ was acquired with four times signal accumulation and its imaging time was $102.4 \mathrm{~s}$. Images were reconstructed as a $256 \times 256$ matrix with the zero-filled interpolation. The resulting nominal planar resolution was $430 \mu \mathrm{m}$. 


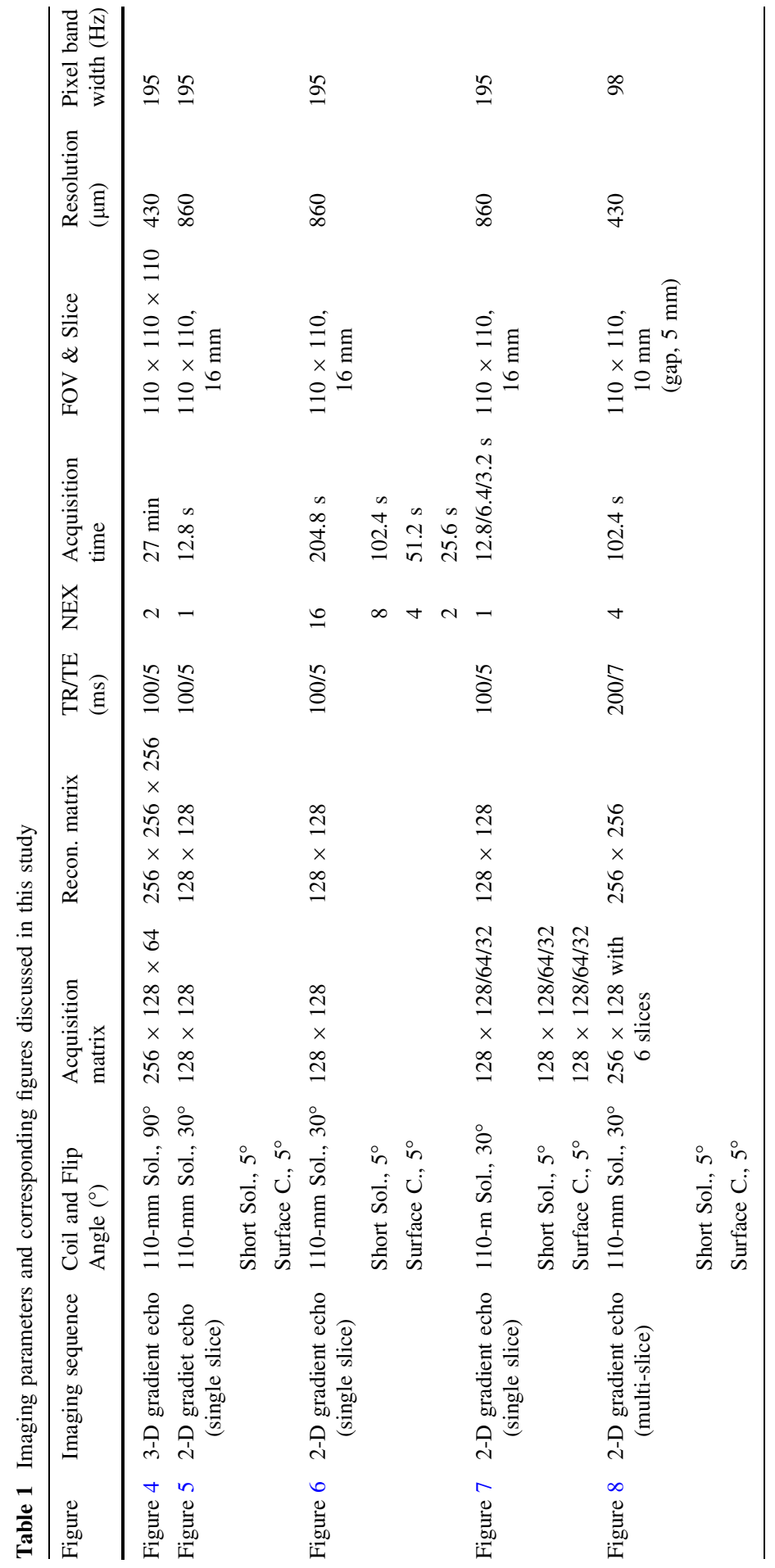



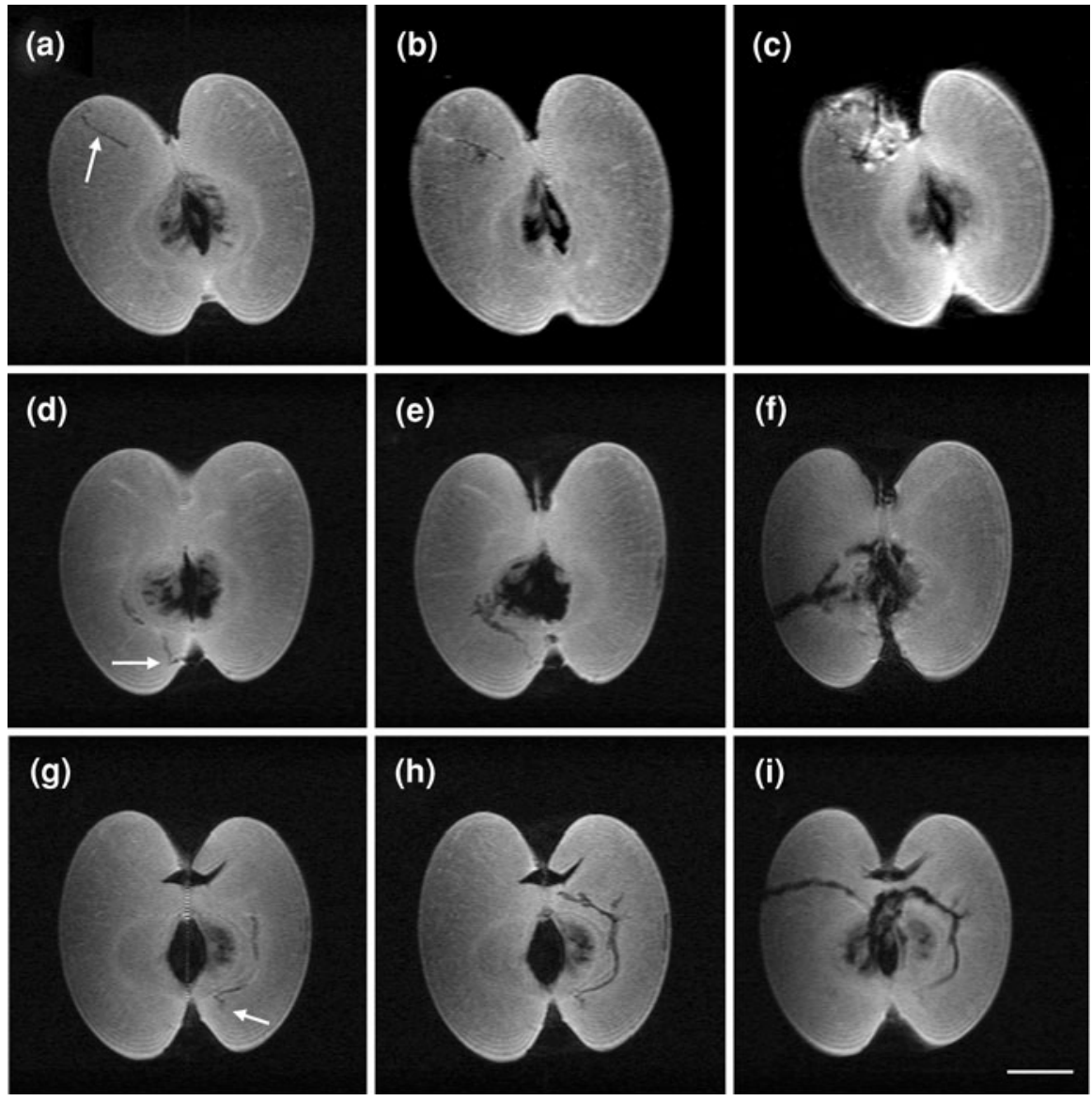

Fig. 4 Tracking the spread of infestation by the 3-D gradient echo method. Fruit with an infested line at an early stage (arrow in a; 7th day), an infested hole (b; 14th day), and large infestation (c; 21st day) at the upper sarcocarp of the fruit. Fruit infested from the bottom left near the calyx (arrow in d; 7th day), with an expanding hole around the coreline vasculature (e; 14th day), and with a passage out from the side of the fruit (f; 21 st day). Fruit infested from the bottom right near the calyx (arrow in $\mathbf{g}$; 7 th day), with an expanding hole around the coreline vasculature (h; 14th day), and the escape tunnel through the left shoulder of the fruit (i; 21st day). Scale bar $20 \mathrm{~mm}$

\section{Results}

\subsection{Trace of Expanding Infestation of Apple Fruits by the 3-D Gradient Echo Method}

Expansion of infested holes in three apples was tracked as shown in Fig. 4. These images were drawn from among the seven fruits into which larvae hatched and entered on 12 December 2008. Measurements were carried out on the 7th day (Fig. 4a, d, g), 14th day (Fig. 4b, e, h), and the 21st day (Fig. 4c, f, i) after hatching. The apples were carefully set at the same position in the long solenoid coil to 

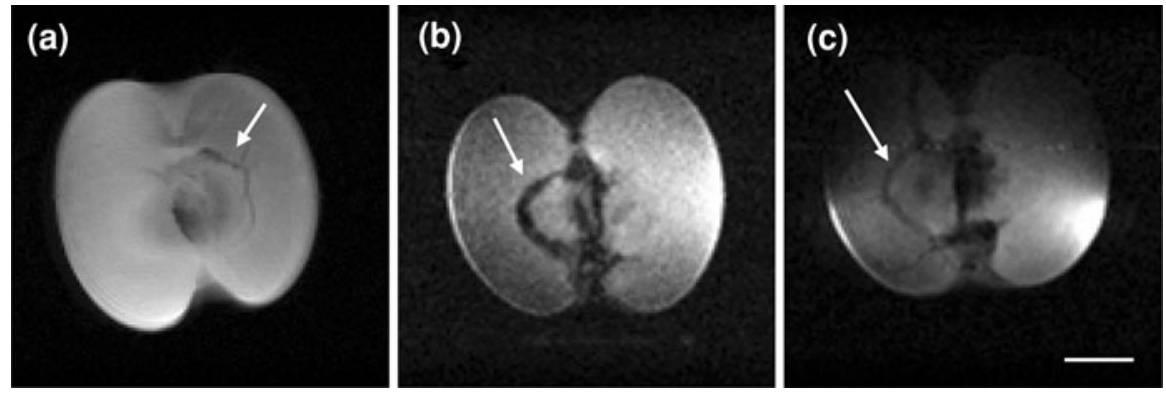

Fig. 5 Vertical slice images acquired by the single-slice 2-D gradient echo method using different detectors. a 110-mm solenoid coil detector with slice direction $\beta$ in Fig. 2 d. b Short solenoid coil detector with slice direction $\delta$ in Fig. 2e. c Surface coil detector with slice direction $\zeta$ in Fig. 2f. Arrows indicate infested holes. These images illustrate different characteristics of the detectors. Scale bar $20 \mathrm{~mm}$
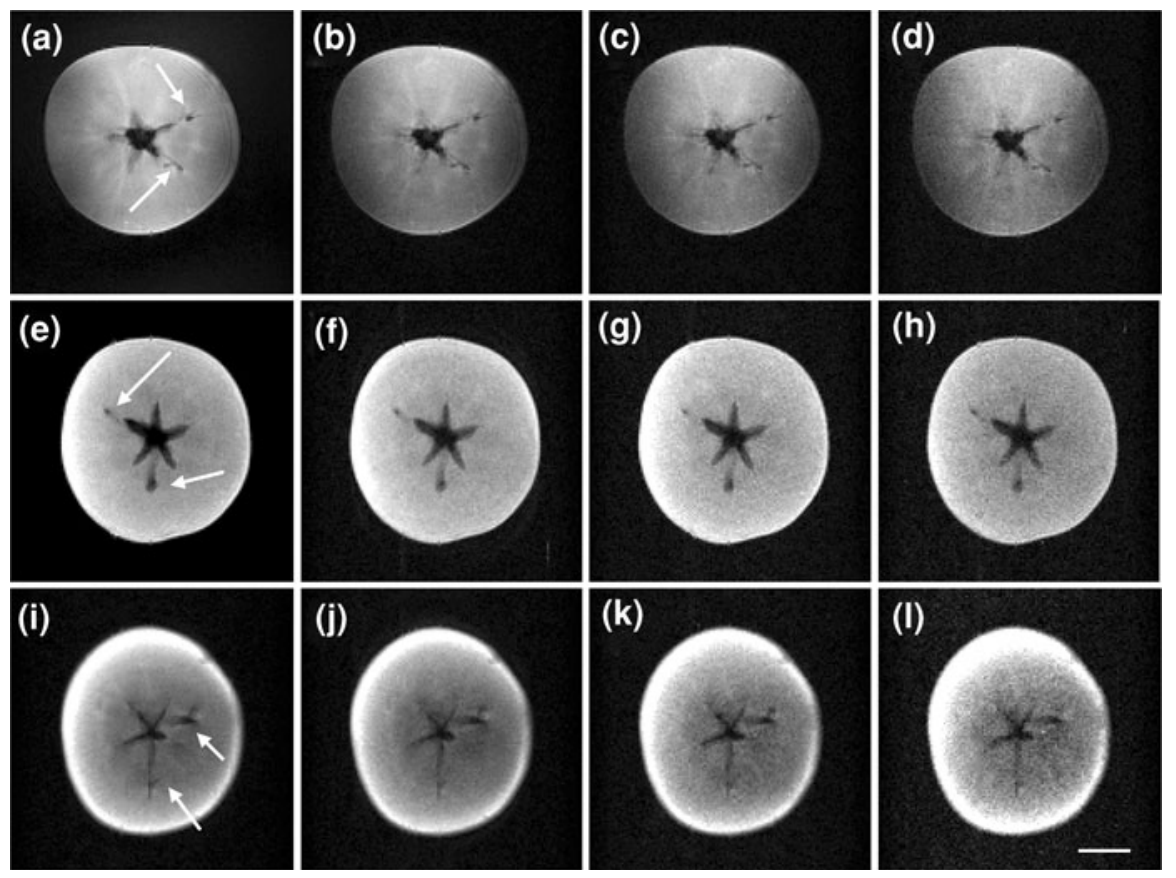

Fig. 6 Effects of accumulation times (16, 8, 4 and 2) on 2-D gradient echo images acquired by different detectors. Images were acquired by a single-slice imaging sequence using a $128 \times 128$ matrix and thickness of $16 \mathrm{~mm}$. The top row (a-d) is from the 110-mm solenoid coil detector. The central row (eh) is from the short solenoid coil detector. The bottom row (i-l) is from the surface coil detector. The first column presents 16 times, the second 8 times, third 4 times and the last 2 times. Arrows indicate infested holes. Scale bar $20 \mathrm{~mm}$

support the 3-D image analysis. The measurements were performed by the 3-D gradient echo method, which returned high-intensity signals. Small infestations were detected and an expansion of the infested hole could be traced in the image 

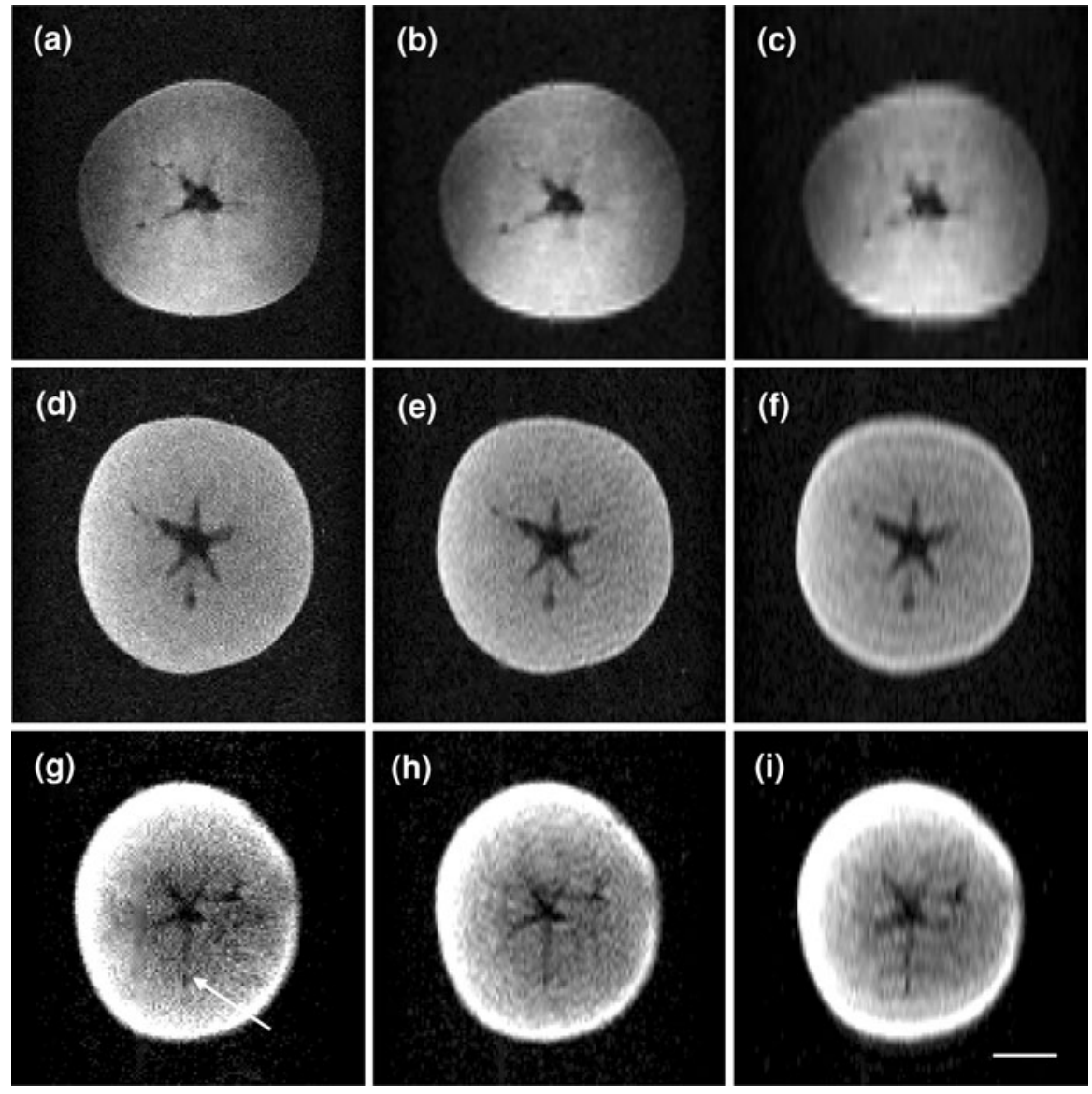

Fig. 7 Effects of phase-encoding steps (128, 64, and 32) on 2-D gradient echo images acquired by different detectors. Images were acquired by a single-slice imaging sequence with $16 \mathrm{~mm}$ slice thickness and reconstructed as $128 \times 128$ matrix. The top row $(\mathbf{a}-\mathbf{c})$ is from the $110-\mathrm{mm}$ solenoid coil detector. The center row $(\mathbf{d}-\mathbf{f})$ is from the short solenoid coil detector. The bottom row $(\mathbf{g}-\mathbf{i})$ is from the surface coil detector. The first column presents 128 steps, the second 64 steps, and the third 32 steps. Scale bar $20 \mathrm{~mm}$

sets of Fig. 4a-c, d-f, or g-i. Positioning accuracy of each apple in the longitudinal study was within $\pm 1 \mathrm{~mm}$, which was greater than the imaging resolution of $430 \mu \mathrm{m}$. However, the 3-D high-resolution acquisition could find the same infestation in the longitudinal images under the limit of 3-D image rotation analysis by software. The single measurement time was $27 \mathrm{~min}$, which was used for tracing the progression of infestation in fruits within 1 day. The 3-D images were rotated to clearly illustrate the infested holes for ecological understanding. Signal intensities of the images were weak (Fig. 3a, $110 \mathrm{~mm} \mathrm{Sol}$.) at the extremities of both sides of the fruits, because imaging slices were determined along the axis of the coil ( $\alpha$ in Fig. 2d). This, however, was not a serious drawback for finding trivial infestation in the fruits. 
Fig. 8 2-D multi-slice gradient echo images $(256 \times 128$ matrix, $\mathrm{TR} / \mathrm{TE}=200 \mathrm{~ms} / 7 \mathrm{~ms}$ ) acquired with a slice thickness of $10 \mathrm{~mm}$ and gaps of $5 \mathrm{~mm}$ by different detectors: a 110-mm solenoid coil detector, b short solenoid coil detector, and c surface coil detector. The flip angles, which were

experimentally adjusted at the coil's center, were $30^{\circ}$ for the $110-\mathrm{mm}$ solenoid coil detector, and $5^{\circ}$ for both of the 2-D specified detectors. Images at the coil's center are indicated as \pm 0 (mm). These images indicate the relationship between the signal intensity and the distance from the slice center. Scale bar $20 \mathrm{~mm}$
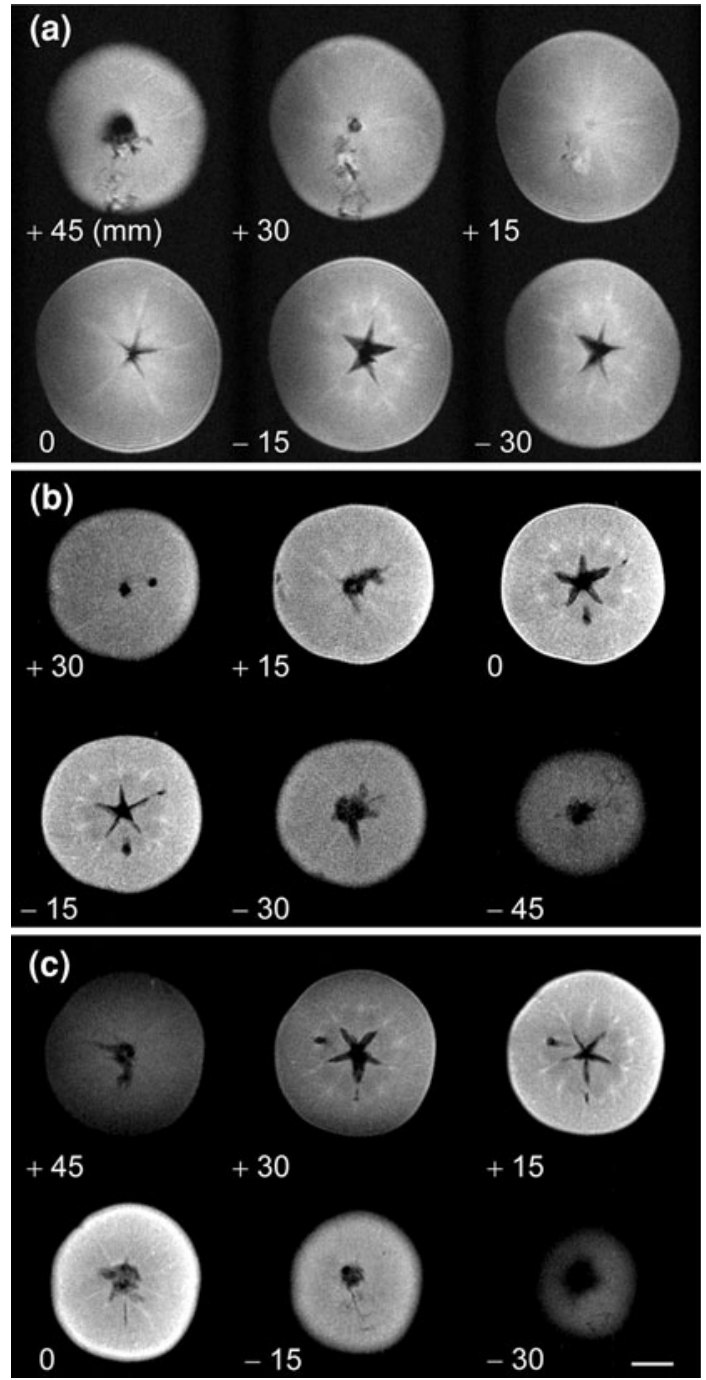

In the fruit depicted on the top row of Fig. 4, a thin line was observed at the top left region of the fruit, opposite to the stem on the 7th day (indicated by arrow in Fig. 4a). The infestation then expanded, and a hole was observed in the upper sarcocarp on the 14th day (Fig. 4b). A large infestation was observed in the left shoulder of the fruit on the 21st day (Fig. 4c). No larva seemed to reach the core of the fruit. In the fruit shown by the central row of images, a larva entered the fruit from the left bottom near the calyx on the 7th day (arrow in Fig. 4d), expanded the infestation along the coreline vasculature until the 14 th day (Fig. 4e), and then made a large passage out of the fruit on the 21 st day (Fig. 4f). In the bottom row, infestation could be seen beginning from the right side of the calyx on the 7th day (arrow in Fig. $4 \mathrm{~g}$ ) and proceeding along the coreline vasculature upward on the 14th day (Fig. 4h). After 
entering the core, the larva made a tunnel through the upper left side of the fruit on the 21 st day (Fig. 4i). In general, larvae tended to enter from near the calyx or shoulder of the fruit and to infest around the vascular bundles of the core.

\subsection{Rapid Detection of Infestation by Single-Slice 2-D Images}

High-sensitivity detectors with a short solenoid coil (Fig. 2b) or a surface coil (Fig. 2c) were devised to shorten the measurement times to suit the requirements for the type of examination used during export management. The results obtained using these detectors were compared with those obtained with the 110-mm solenoid coil detector used for 3-D measurements (Fig. 2a).

Figure 5 presents the vertical images obtained using the three different types of detectors by the single-slice 2-D gradient echo method (16-mm slice thickness). The slice section directions were designated as $\beta$ for the $110-\mathrm{mm}$ solenoid coil detector (Fig. 2d), $\delta$ for the short solenoid coil detector (Fig. 2e), and $\zeta$ for the surface coil detector (Fig. 2f). The images indicate infestation holes around the core (arrows), where larvae moved along the coreline vascular bundles among the carpels. As well as the spatial signal profiles shown in Fig. 3a and b, the image obtained using the $110-\mathrm{mm}$ solenoid coil detector exhibited rather uniform signals on the image (Fig. 5a), and the two high-sensitivity 2-D detectors displayed different characteristics. Signal intensity in the central area of the fruit along the coil axis ( $\delta$ in Fig. 2e) was strong, while that in both the top and lower sides of the fruit was small (Fig. 5b). A similar tendency was observed for the images from the 110-mm solenoid coil detector, if the slice plain was set along the coil axis ( $\alpha$ in Fig. $2 d)$; signal intensities declined at the edges of the coil (Fig. 4). As for the surface coil detector, signals that were strong near the coil surface rapidly declined with distance from the circuit in the coil axis ( $\zeta$ in Figs. 2 f, $3 \mathrm{a}, 5 \mathrm{c}$ ).

The primary factor that influenced the measurement time was the number of signal accumulations, which in turn influenced the signal-to-noise ratio. Changes associated with the decrease of accumulation times from 16 to 2 on the horizontal images by the single-slice 2-D gradient echo method $(128 \times 128$ matrix, $16 \mathrm{~mm}$ slice thickness) are depicted in Fig. 6. The changes in the signal-to-noise ratios versus the number of accumulations resulted well according to the measurement theory of averaging. The top row in Fig. 6 indicates the images from the $110-\mathrm{mm}$ solenoid coil detector, the central row the images from the short solenoid coil detector, and the bottom row the images from the surface coil detector. However, although image clarity was diminished by reducing accumulation times, infestations could be discerned on images obtained with as few as two accumulation times for all detectors.

The second factor concerning the measurement time was the number of 2-D phaseencoding steps (128, 64, and 32) without signal averaging. The 64 and 32 phaseencoding step images were reconstructed as $128 \times 128$ matrix images. A reduction of phase-encoding steps resulted in shorter measurement times. Figure 7 illustrates the effect of phase-encoding steps examined by fixing the readout sampling number of 128 points. Infested holes were not obvious on the images with 128 phase-encoding steps acquired by the $110-\mathrm{mm}$ solenoid coil detector (Fig. 7a) and not discriminated 
by the surface coil detector (Fig. $7 \mathrm{~g}$ ), while they were clearly detectable by the short solenoid coil detector (Fig. 7d). Image resolutions in the 2-D phase direction declined further, as phase-encoding steps decreased for the 110-mm solenoid coil (Fig. 7b), the surface coil (Fig. 7h) and the short solenoid coil (Fig. 7e) detectors. The images (Fig. $7 \mathrm{~g}-\mathrm{i}$ ) acquired with the surface coil presented a ring-shaped strong signal pattern around the apple surface and this was caused by a combination of the spatial grading detection property of the surface coil shown in Fig. $3 \mathrm{~b}$ and its slice thickness of $16 \mathrm{~mm}$, but came from the effect of an apple's surface shape. Because Fig. 7d-f has a flat intensity gradation in image planes as compared with that of Fig. $7 \mathrm{a}-\mathrm{c}, \mathrm{g}-\mathrm{i}$, the short solenoid coil detector provides the most reliable apple images for the larvae and larvae hole detection. These results indicate that the 2-D image acquisition of the gradient echo methods with the short solenoid coil with 64 or 32 phase-encoding steps (6.4 or $3.2 \mathrm{~s}$ ) was the best for the purpose of this study.

\subsection{Rapid Detection of Infestation by Multi-slice 2-D Images}

Next, horizontal images were obtained using the multi-slice 2-D gradient echo method (Fig. 8). Based on the measurement conditions providing satisfactory quality images for all detectors as in Fig. 6, four accumulations in each phase encoding were acquired with a $256 \times 128$ matrix for six slices of $10 \mathrm{~mm}$ thickness and $5 \mathrm{~mm}$ gaps in an FOV of $11 \times 11 \mathrm{~cm}$. TR and TE were 200 and $7 \mathrm{~ms}$, and flip angles $\left(110-\mathrm{mm}\right.$ Sol. $=30^{\circ}$, Short Sol. $=5^{\circ}$, and Surface Coil $\left.=5^{\circ}\right)$ were adjusted experimentally at the coil's centers. It took $102.4 \mathrm{~s}$ to image a set of six slices. The images obtained by the 110-mm solenoid coil detector (Fig. 8a) showed constant image resolution along the slice positions compared with the other 2-D-specified detectors (Fig. 8b, c). The 2-D detectors were highly sensitive and provided clear contrast of pericarp tissues and infested holes on the slices near the coil's centers. Figure 8 shows that the short solenoid coil is able to acquire the usable 2-D image slices for apple with the 2-D multi-slice imaging within $\pm 45 \mathrm{~mm}$ from the coil center, while the surface coil has a narrow sensitive volume within $\pm 30 \mathrm{~mm}$. Signal intensity gradually declined with distance from the center of the coil for the short solenoid coil detector (Fig. 8b), and it declined severely with distance from the circuit plate for the surface coil detector (Fig. 8c).

\section{Discussion}

For the purpose of plant protection associated with the export of fruits, noninvasive detection of infestation of apple fruits by the peach fruit moth is very much required. There are apparatuses using the absorption of near-infrared light to detect infestation in fruits. These devices are, however, reported to misclassify sound fruits as infested ones with a probability of $40 \%$ [19]. MRI is a practical means for studying insects in their habitat or normal living circumstances [7-12]. The drawback of MRI for detecting infestation by insects in production management is the fact that the current apparatuses are large, heavy, expensive, difficult to maintain and have a high running cost. This is also an obstacle preventing MRI from being used in the 
development of other applications in ecological research. In this regard, small, dedicated MRI devices equipped with permanent magnets are thought to overcome a part of this drawback.

We have devised a sensitive and lightweight apparatus with a large measurement cell applicable for grown fruits. $\mathrm{Nd}-\mathrm{Fe}-\mathrm{B}$ blocks generating high magnetic field strength were used to construct an U-shaped permanent magnet with a wide air gap (160 mm) (NEOMAX Engineering Co. Ltd., Gunma, Japan). Consequently, the magnetic field could be increased to $0.2 \mathrm{~T}$ while still meeting the load-bearing requirements of an ordinary concrete building $\left(400 \mathrm{~kg} / \mathrm{m}^{2}\right)$ (Fig. 1a, b) in an ordinary research room or an inspecting office close to fields. This apparatus was capable of detecting growing or grown larvae in fruits by the 3-D spin echo method if a rather long measurement time ( $82 \mathrm{~min})$ was acceptable [16]. The slow $T_{2}$ decay of signals in a low magnetic field [20] might be favorable to obtain good-quality images for materials with nonhomogeneous susceptibility, such as apple fruits, in spite of the comparatively low sensitivity and limited resolution of the apparatus. Moreover, fairly small infestations by larvae at early stages of growth were detected and the movements of larvae could be tracked through the expansion of infested holes by the 3-D gradient echo method with a 27 min measurement time (Fig. 4). This scope may satisfy the spatial and time resolution requirements for tracing the expansion of infested holes and elucidating larval actions in fruits using a large number of samples.

Another disadvantage of MRI is that it requires long measurement times because its sensitivity is low compared with optical and molecular biological methods. The issue of measurement time has severely restricted the development of applications using MRI. We sought to shorten the measurement time, and investigated the possibility of using the 0.2 -T MRI apparatus as a means for surveying damage by the insect and, further, as a sensor for an automated system for detecting infested fruits from the standpoint of protecting natural ecological systems in overseas trade. 3-D measurement was not suitable for shortening measurement times due to a large number of phase-encoding steps based on the principles of MRI and, therefore, 2-D measurement was attempted. Measurements were carried out by the fast low-angle shot (FLASH) method [21], or the fast 2-D gradient echo method using small flip angles, which provided the strongest signals on the echo monitor. Here, however, the sensitive detector used to obtain 3-D image data (Fig. 2a) did not exhibit a satisfactory image quality (Fig. 7, the top line) as in the 3-D images when the measurement time was shorter than $1 \mathrm{~min}$. Therefore, two other detectors specified for 2-D measurement were designed based on the results of Hoult and Richards [18]. One system employed a short solenoid coil detector with the diameter reduced to the size of the fruit $(94 \mathrm{~mm})$ and the coil length minimized to $35 \mathrm{~mm}$ (Fig. 2b, e). Decreasing the coil diameter or the coil length improved the signal-to-noise ratio (Fig. 3a, b) and the image quality (Fig. 7d-f); infested holes could be detected by a single transient acquisition with a $128 \times 64$ matrix at a rate of $6.4 \mathrm{~s}$ per image (Fig. 7e). The other system employed a surface coil detector (Fig. 2c). The coil was designed using four loops made of two printed copper boards with double circuits, 
and the inner diameter of the coil $(75 \mathrm{~mm})$ was less than that of the fruit (Fig. $2 \mathrm{f}$ ). The layout was thought to be suitable for strong induction and enabled the detector to achieve high sensitivity near the circuit boards. However, the image obtained by this detector was not as discriminating as the short solenoid detector, and was estimated to require $12.8 \mathrm{~s}$ (Fig. $7 \mathrm{~g}$ ) or more to clearly delineate infestation (Fig. 61).

These detectors devised specifically for 2-D measurement were more sensitive compared with that used for 3-D measurement (Figs. 6, 7), but the signal intensity decreased quickly when the slice planes moved away from the center or the surface of the coil (Fig. 8). This tendency (Fig. 3a, b) was greater for the surface coil detector (Fig. 5c) than that for the short solenoid detector (Fig. 5b). Considering the clarity of images and the slow gradient of signal decline, the short solenoid coil was determined to be preferable for an automated selection system: if several detectors were placed in series and images sequentially taken in different slice planes, infestation occurring in a whole fruit could be measured within a time as short as $6.4 \mathrm{~s}$ (Fig. 7e). At present, however, this proposal requiring multi-detectors is not practicable. But the surface coil detector, as well as the short solenoid coil detector, provided sharp and discriminative images by the multi-slice method (Fig. 8c) in the acquisition time of $102.4 \mathrm{~s}$. The multi-slice imaging method in an FOV of $11 \times 11 \mathrm{~cm}$ with six to eight images with a slice thickness of $10 \mathrm{~mm}$ and slice gaps of $5 \mathrm{~mm}$ might miss small infestations in the gaps. However, by using two duplicated imaging measurements, covering the remaining slice gaps in the time series requires just a doubled imaging time of $204.8 \mathrm{~s}$.

To date, many ecological discoveries have been studied by MRI: the oxygen diffusion barrier between the root cortex and the soybean nodules has been delineated [22, 23]; the observation that only bound water is found in winterdormant flower buds, but free water is detected in summer-dormant or eco-dormant buds $[24,25]$; tracing the cavitation and embolism leading to the decline of xylem function in the tree plants that suffered from pathogens [26] and water stress [27]; and visualization of water flow in xylem and phloem in plant stems [28, 29]. These studies, carried out using MRI with high magnetic field strengths, have suffered from restricted possibilities due to functional and structural operating characteristics of the given apparatus that often disrupted the relationship between the materials observed and the circumstances of their normal habitat. Recently, in situ measurements using small magnets of low magnetic fields were reported for analysis of cavitation in tree stems infected by pine wilt disease [30] and for water transport in xylem and phloem [31], where there remained the difficulty that receiver coils had to be repeatedly wound around the stems. In this regard, the lightweight magnet of $0.2-\mathrm{T}$ field strength with an open structure and sensitive handmade detectors using the common noise-shielding housing in the current investigation alleviate such problems, common to MRI measurements, and provide a means for inspecting the events occurring in an intact plant, such as infestation by larvae in a fruit attached to the mother tree. Thus, we are convinced that the $0.2-\mathrm{T}$ MRI apparatus will be helpful for exploiting new ideas for research on the protection and preservation of the natural environment. 


\section{Conclusions}

We have proven that the 0.2-T MRI apparatus is beneficial to ecological studies of larval activity in grown fruits as well as harvested fruits, as reported previously [16]. Larvae frequently entered the fruit near the calyx and tended to infest around the coreline vasculature where sugars accumulate. These larval functions are to be investigated further in relation to the growth stages of the fruit, strains of larvae and environmental circumstances that influence the movements and actions of the insect. Infestation in the single-slice 2 -D gradient echo method $(128 \times 64$ matrix, $16 \mathrm{~mm}$ thickness) was detected in $6.4 \mathrm{~s}$, and image acquisition time may be further reduced by properly tuning the apparatus if the infested holes are larger than $1 \mathrm{~mm}$, the resolution of the images $(860 \mu \mathrm{m})$. The multi-slice 2-D gradient echo measurement $(256 \times 128$ matrix, $10 \mathrm{~mm}$ slice thickness, $430 \mu \mathrm{m}$ in plane resolution) is useful for examining the infestation in a whole fruit when short time is required. This apparatus has the potential to serve as a means of examining infested conditions in fruits or as a sensor for an automated system for detecting infested fruits as required for plant protection and preservation of the natural ecological system, by using 2-D specified detectors with increased sensitivity. Remaining research challenges include how the infested holes occurring in the 3-D space of a fruit can be completely detected by a stack of 2-D images and what slice thickness would be the most appropriate to detect infestations.

Open Access This article is distributed under the terms of the Creative Commons Attribution Noncommercial License which permits any noncommercial use, distribution, and reproduction in any medium, provided the original author(s) and source are credited.

\section{References}

1. H. Narita, Bull. Akita Fruit-Tree Expt. Sta. 17, 31-128 (1986) (in Japanese with English summary)

2. Y. Ishiguri, S. Toyoshima, Appl. Entomol. Zool. 41, 685-690 (2006)

3. S.Y. Wang, P.C. Wang, M. Faust, Sci. Hortic. 35, 227-234 (1988)

4. C.J. Clark, J.S. MacFall, R.L. Bieleski, Sci. Hortic. 73, 213-227 (1998)

5. P. Barreiro, A. Moya, E. Correa, M. Ruiz-Altisent, M. Fernández-Valle, A. Peirs, K.M. Wright, B.P. Hills, Appl. Magn. Reson. 22, 387-400 (2002)

6. M.J. McCarthy, B. Zion, P. Chen, S. Ablett, A.H. Darke, P.J. Lillford, J. Sci. Food Agric. 67, 13-20 (1995)

7. G. Gassner, J.A.B. Lohman, Proc. Natl. Acad. Sci. USA 84, 5297-5300 (1987)

8. B.A. Goodman, S.C. Gordon, J.A. Chudek, G. Hunter, J.A.T. Woodford, J. Insect Physiol. 41, 419-424 (1995)

9. S. Wecker, T. Hörnschemeyer, M. Hoehn, Magn. Reson. Imaging 20, 105-111 (2002)

10. F.L.W. Ratnieks, J. Insect. Sci. 3(5), online: http://insectscience.org/3.5 1-9 (2003)

11. J.A. Chudek, G. Hunter, R.L. MacKay, S. Moritz, A.N.E. Birch, I.E. Geoghegan, R.J. McNicol, M.E.N. Majerus, in Spatially Resolved Magnetic Resonance: Methods, Materials, Medicine, Biology, Rheology, Geology, Ecology, Hardware, ed. by P. Blümler, B. Blümich, R. Botto, E. Fukushima (Wiley-VCH, Weinheim, 1998), pp. 467-471

12. K.J. Hallock, J. Insect Sci. 8(10) available online: http://insectscience.org/8.10 1-7 (2008)

13. P.T. Callaghan, Principles of Nuclear Magnetic Resonance Microscopy (Clarendon Press, Oxford, 1991)

14. F. Ihara, K. Yaginuma, N. Ishida, M. Koizumi, Jpn. J. Appl. Entomol. Zool. 52, 123-128 (2008) (in Japanese with English abstract) 
15. M. Koizumi, S. Naito, T. Haishi, S. Utsuzawa, N. Ishida, H. Kano, Magn. Reson. Imaging 24, 1111-1119 (2006)

16. T. Haishi, H. Koizumi, T. Arai, M. Koizumi, H. Kano, Jpn. J. Ecol. 59, 249-257 (2009) (in Japanese with English abstract)

17. T. Haishi, T. Uematsu, Y. Matsuda, K. Kose, Magn. Reson. Imaging 19, 875-880 (2001)

18. D.I. Hoult, R.E. Richards, J. Magn. Reson. 24, 71-85 (1976)

19. S. Toyoshima, Y. Nakamoto, M. Takanashi, T. Masuda, Bull. Natl. Inst. Fruit Tree Sci. 5, 87-94 (2006)

20. M. Koizumi, S. Naito, H. Kano, T. Haishi, Nippon Shokuhin Kagaku Kogaku Kaishi 56, 146-154 (2009) (in Japanese with English abstract)

21. A. Haase, J. Frahm, D. Matthaei, W. Hänicke, K.-D. Merboldt, J. Magn. Reson. 67, 258-266 (1986)

22. J.S. MacFall, P.E. Pfeffer, D.B. Rolin, J.R. MacFall, G.A. Johnson, Plant Physiol. 100, 1691-1697 (1992)

23. J.A. Chudek, G. Hunter, J.I. Sprent, G. Wurz, Magn. Reson. Imaging 15, 361-368 (1997)

24. M. Faust, D. Liu, M.M. Millard, G.W. Stutte, HortScience 26, 887-890 (1991)

25. A. Erez, M. Faust, M.J. Line, Sci. Hortic. 73, 111-123 (1998)

26. A.G. Pérez-Donoso, L.C. Greve, J.H. Walton, K.A. Shackel, J.M. Labavitch, Plant Physiol. 143, 1024-1036 (2007)

27. M.J. Clearwater, C.J. Clark, Plant Cell Environ. 26, 1205-1214 (2003)

28. W. Köckenberger, J.M. Pope, Y. Xia, K.R. Jeffrey, E. Komor, P.T. Callaghan, Planta 201, 53-63 (1997)

29. A.D. Peuke, M. Rokitta, U. Zimmermann, L. Schreiber, A. Haase, Plant Cell Environ. 24, 491-503 (2001)

30. K. Fukuda, S. Utsuzawa, D. Sakaue, Tree Physiol. 27, 969-976 (2007)

31. C.W. Windt, F.J. Vergeldt, P.A. Jager, H. Van As, Plant Cell Environ. 29, 1715-1729 (2006) 